\title{
DMAEMA-grafted cellulose as an imprinted adsorbent for the selective adsorption of 4-nitrophenol
}

\author{
Daning Lang $\cdot$ Ming Shi $\cdot$ Xia Xu $\cdot$ Shixue He $\cdot$ Chao Yang $\cdot$ Lu Wang \\ Ronglan Wu $\cdot$ Wei Wang $(\mathbb{D} \cdot$ Jide Wang
}

Received: 29 November 2020/ Accepted: 5 May 2021/Published online: 25 May 2021

(C) The Author(s) 2021

\begin{abstract}
Nitrophenol is a highly toxic environmental pollutant. It is a challenge to selectively remove it from a mixture of various pollutants. Herein, we report a study on the selective adsorption of 4-nitrophenol by using molecularly imprinted polymers (MIPs). The imprinted polymer was synthesized using cellulose as a framework, onto which, the complex of the imprinting molecule (i.e., 4-nitrophenol) and a candidate material [namely, 2-(dimethylamino)ethyl methacrylate. DMAEMA] was grafted. The obtained MIP showed an excellent adsorption capacity with good selectivity. Also, the adsorption of 4-nitrophenol by the obtained MIP was fast and the adsorbent exhibited good recyclability. The thermodynamics and kinetics of the adsorption process of 4-nitrophenol by MIP was thoroughly studied, where an otherwise-equivalent non-imprinted
\end{abstract}

polymer was used as a control in the experiments. The selectivity of the MIP adsorbent for 4-niteophenol was evaluated by two types of experiments: (1) adsorption experiments in single-component adsorbate systems (containing 4-nitrophenol, 3-nitrophenol, catechol, or hydroquinone), and (2) competitive adsorption experiments in binary adsorbate systems (containing 4-nitrophenol plus either 3-nitrophenol, catechol or hydroquinone). The selectivity coefficient for 4-nitrophenols was twice of those of other phenols (that were all around 2), indicative of the extent of the affinity of MIPs to these phenolic compounds. The recyclability of the adsorbent was evaluated for 5 adsorptiondesorption cycles, where the adsorption capacity of the last cycle remained over $90.2 \%$ of that of the first cycle.
D. Lang $\cdot$ M. Shi $\cdot$ X. Xu $\cdot$ S. He

C. Yang $\cdot$ L. Wang $\cdot$ R. Wu $(\bowtie) \cdot$ J. Wang

Key Laboratory of Oil and Gas Fine Chemicals, School of

Chemical Engineering, Xinjiang University,

Urumqi 830046, China

e-mail: ronglanwu@163.com

W. Wang $(\bowtie)$

Institute of Chemistry and Center for Pharmacy,

University of Bergen, 5020 Bergen, Norway

e-mail: wei.wang@uib.no 


\section{Graphic Abstract}

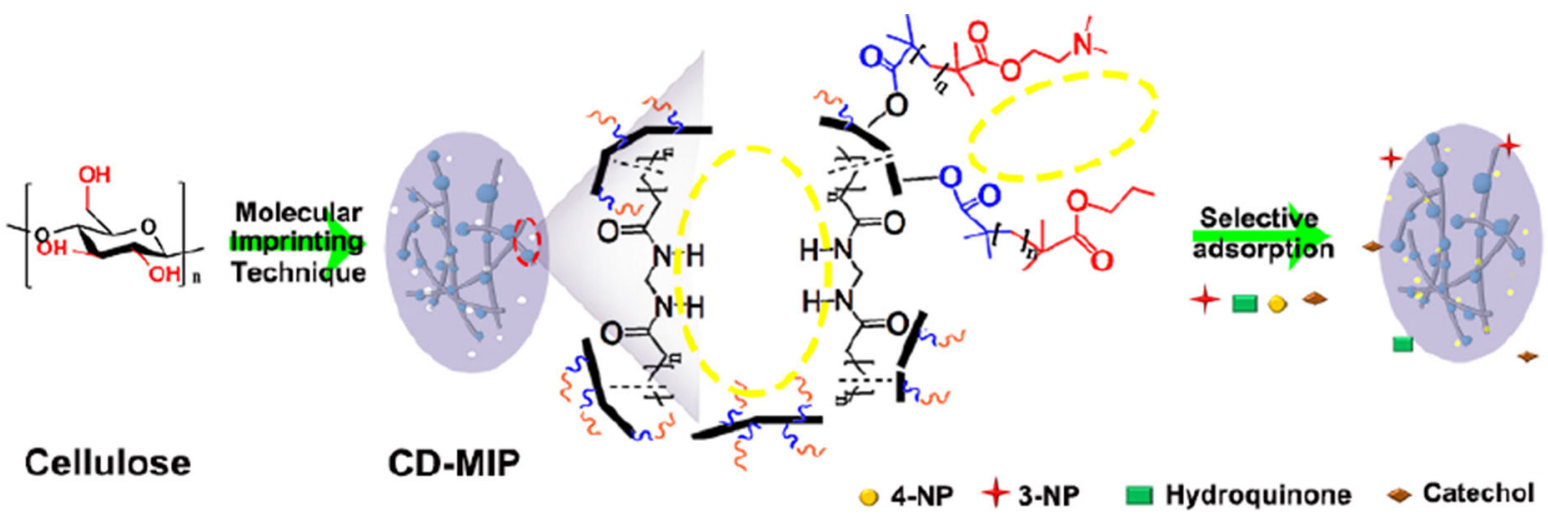

Keywords Molecular imprinting polymer . Adsorption · Cellulose · DMAEMA · Wastewater treatment

\section{Introduction}

The phenolic compound 4-nitrophenol, is commonly used as a raw material in various aspects of the chemical industry, e.g., in the production of dyes, pharmaceutics, and pesticides. Examples of its application in the production of pharmaceutics include its use as an intermediate in the synthesis of paracetamol and as a precursor in the preparation of phenetidine and acetophenetidin (Jiang et al. 2016; Pan et al. 2007). Due to its irritancy, corrosively and toxicity, the 4-nitrophenol found in the wastewater of chemical industries is deemed an environmental pollutant that should be removed before discharging the sewage to the environment (Koyuncu et al. 2011; Mehdinia et al. 2014).

So far, many methods have been applied for removing 4-nitrophenol from the wastewater, including adsorption (Rong and Han 2019), extraction (Gulati et al. 2020), photocatalytic degradation, and biological methods. For example, Zhang et al. found a good adsorption capacity for 4-nitrophenol in the magnetite that was modified with tetraethoxysilane and vinyltriethoxysilane (Zhang et al. 2019). Ma et al. (2019) used biocarbon prepared from leaves of Platycladus Orientalis as absorbent for 4-nitrophenol, where a maximum adsorption capacity of $622.73 \mathrm{mg} / \mathrm{g}$ (at $298 \mathrm{~K}$ ) was reported. Meng et al. used $\mathrm{N}$-isopropylacrylamide as a functional monomer, which was grafted on cyclodextrin/azobenzene to prepare a light-responsive adsorbent for 4-nitrophenol. The adsorbent could adsorb 4-nitrophenol at $430 \mathrm{~nm}$ at $20{ }^{\circ} \mathrm{C}$ and release it at $40{ }^{\circ} \mathrm{C}$ (Meng et al. 2020). Liang et al. (2020) synthesized $\mathrm{Fe}_{3} \mathrm{O}_{4} @$ $\mathrm{SiO}_{2} @$ PNP-SMIP surface imprinted polymer and used solid-phase extraction to remove 4-nitrophenol. Alhokbany et al. used chitosan as a carbon source to prepare porous Ag particles doped with nitrogen. Under visible light irradiation for $40 \mathrm{~min}$, 98\% of 4-nitrophenol was reduced to 4-aminophenol (Alhokbany et al. 2019). Lu et al. (2020) reported a study on degradation of 4-nitrophenol by treating acclimated activated sludge with ultrasound.

Among methods applied for removing 4-nitrophenol from solutions, the adsorption method stands out due to its many advantages, such as being operationally simple, exhibiting a high removal rate, and being easy to translate the process into industrial applications. Such advantages stand out against the shortcomings of other methods, such as (1) the toxicity of the extraction liquid in the extraction method, as well the unstable character of this method, (2) the large energy consumption, high costs and low removal rates encountered in the chemical method, and (3) the induced self-poisoning of microorganisms and the difficulties faced in the degradation of pollutants in the biological method. Nevertheless, the efficiency of the adsorption process is highly dependent on the adsorbents (Bahrami et al. 2020). Thus, there is a strong 
demand for developing new low-cost adsorbents, featuring high adsorption capacity, high selectivity, and good recyclability.

Cellulose is a biopolymer abundantly found in nature. It is a low cost, renewable, biodegradable, nontoxic, and environmentally harmless material, which is widely used in wastewater treatment, despite the fact that its adsorption capacity in its untreated form is limited (Kumari and Chauhan 2014; Velempini et al. 2019). Thus, it is common to adopt the approach of chemical modification for improving the adsorption capacity of cellulose. The chemical modification of cellulose is not restricted to increasing its adsorption capacity, and imparting cellulose with new properties is also desirable. Examples of such properties include selective adsorption (Cao et al. 2018; Liu et al. 2015), bi-functionality (Gericke et al. 2013; Peydayesh et al. 2020Ronglan Wu et al. 2015), and photo-degradability (Belhouchet et al. 2019; Li et al. 2020; Mei et al. 2019; Wu et al. 2015; Xiaolin Man et al. 2015).

Molecular imprinting technology (MIT) is a mature and effective molecular recognition technology, which operates in analogy with the recognition mode of lock and key found in enzymes. The technology has been used to prepare polymers [called molecularly imprinted polymers (MIPs)] with specific capabilities for selective recognition for template molecules (Cantarella et al. 2019; Pichon et al. 2019; Yoshikawa et al. 2016). MIPs offer many advantages, including ease of synthesis, good chemical stability, and reusability, which have prompted their application in some scientific and technical fields, such as chromatography, antibody mimics (Schirhagl 2014), sensors (Cui et al. 2020; Kenawy et al. 2018), and drug release systems (Cegłowski et al. 2019). Nevertheless, the applications of MIPs suffer some limitations, due of their high costs, low binding capacity, incomplete removal of the template molecules, and poor adsorption capacity. This means that there is a large unexplored space in the development of new materials by MIT (Wu et al. 2020). Tian et al. (2018) graphed $\mathrm{N}$-isopropylacrylamide on the $2 \mathrm{D}$ graphene oxide to prepare imprinted polymers with good selective adsorption properties for 4-nitrophenol, where an adsorption capacity of $19.78 \mathrm{mg} / \mathrm{g}$ (at $\mathrm{pH}=3$ ) was reported. Due to the good selectivity of MIPs for their template, MIT has also been applied for the preparation of imprinted cellulosic materials. Zhang et al. (2019) successfully introduced silica nanoparticles onto the surface of regenerated cellulose membranes to prepare a new type of molecularly imprinted membrane, which was successfully applied for the separation of paclitaxels. Tan et al. combined regenerated cellulose from cotton with iron oxide nanoparticles by emulsion polymerization, and used 4-nitrophenol as template to prepare a magnetic imprinted porous foam for the selective removal of 4-nitrophenol in water, where a good 4-nitrophenol recycling performance was observed (Tan et al. 2020). These examples exhibit a common approach for synthesis of MIPs by using a framework structure modified by the complex of print molecules and candidate materials. Polymerization of candidate materials constructs recognition sites in the framework and imparts its molecular memory in the polymer matrix. The affinity to the print molecules is highly dependent on the candidate materials. Thus, finding viable candidate materials can significantly improve the properties of MIPs.

In this study, we explored the possibility of using 2-(dimethylamino)ethyl methacrylate (DMAEMA) as the candidate material and grafting it onto cellulose to synthesize a 4-nitrophenol-imprinted adsorbent. The amine group of DMAEMA changes its protonation state with variations in $\mathrm{pH}$, thus affecting the solubility of poly(DMAEMA). This property could be utilized for adjusting the affinity of the polymer for 4-nitrophenol and facilitating both the adsorption and the desorption of 4-nitrophenol by adjusting $\mathrm{pH}$. These ideas was successfully implemented in our study. The imprinted polymer cellulose-g-DMAEMA (CD-MIP), showed excellent adsorption capacity and selectivity. The thermodynamics and kinetics aspects of the adsorption of 4-nitrophenol were thoroughly studied. Furthermore, the recyclability of CD-MIP was evaluated for five adsorption-desorption cycles by adjusting the $\mathrm{pH}$ value of the solution.

\section{Materials and Methods}

Materials

Cellulose was purchased from Hebei Balinway Superfine Materials Co., Ltd. 1-Allyl-methylimidazolium chloride (AmimCl) was purchased from Moni Mooney Chemical Engineering Science \& Technology Co., Ltd. 2-Bromo-2-methylpropionyl bromide, 
2-(dimethylamino) ethyl methacrylate (DMAEMA) and $\quad N, N, N^{\prime}, N^{\prime \prime}, N^{\prime \prime}$-pentamethyldiethylenetriamine (PMDETA) were purchased from Aladdin Reagent Co., Ltd. Cuprous(I) bromide $(\mathrm{CuBr})$ was purchased from Across Chemical Co., Ltd; N,N-methylene-bisacrylamide (MBA), ammonium persulfate (APS) and solvents were purchased from Tianjin Hongyan Reagent Factory. Nitrogen (99.99\%) was purchased from Xinjiang Xintianyi Co., Ltd. Deionized water was used in all experiments. DMAEMA was passed through a basic alumina column to remove the inhibitor before its use.

Synthesis of Cellulose Bromide

Microspheres (Cellulose-Br)

The synthetic route was based on a previous report (Pang et al. 2016). Cellulose (1.0 g) was dispersed in $\mathrm{AmimCl}(19.0 \mathrm{~g})$. The mixture was warmed up to $80{ }^{\circ} \mathrm{C}$ and stirred until cellulose was completely dissolved. The solution of cellulose $(5 \mathrm{wt} \%)$ was transparent. Afterwards, DMF $(50 \mathrm{~mL})$ as a diluent was added into the cellulose solution and stirring was applied to obtain a yellow transparent solution. The solution was first cooled down to room temperature, then put into an ice/water bath until temperature dropped to $0{ }^{\circ} \mathrm{C}$. The solution was purged with $\mathrm{N}_{2}$, and then 2-bromo-2-methylpropionyl bromide ( $8 \mathrm{~mL}$ ) was added dropwise. The mixture was stirred in ice/water bath for $1 \mathrm{~h}$, then warmed up to $50{ }^{\circ} \mathrm{C}$. The temperature was maintained at $50{ }^{\circ} \mathrm{C}$ for $24 \mathrm{~h}$ until the reaction was complete. The product precipitated by adding water, and then it was filtered. The filtrate was collected and dried under vacuum at $50{ }^{\circ} \mathrm{C}$ for $24 \mathrm{~h}$.

\section{Synthesis of Cellulose-g-DMAEMA Based}

Molecularly Imprinted Polymer (CD-MIP)

The synthetic route for CD-MIP can be divided into 4 steps. In step 1, CuBr (7.5 mg), PMDETA (43.3 mg), and ascorbic acid $(9.5 \mathrm{mg})$ were mixed in a $100 \mathrm{~mL}$ volumetric flask and then calibrated with DMF. The molar ratio of the reactants was 1:5:1 (CuBr: PMDETA: ascorbic acid). In step 2, cellulose (50 mg) was mixed with $3.75 \mathrm{~mL}$ of DMF. Afterwards, the dissolved cellulose was mixed with DMAEMA (1.5 g), 4-nitrophenol (5 mg), and MBA $(0.5 \mathrm{~g})$. After the reactants were completely dissolved, APS (10 mg) was added to the mixture. The solution was deoxygenated by purging with $\mathrm{N}_{2}$ for 30 min. Afterwards, the solution was sealed, warmed up to $60{ }^{\circ} \mathrm{C}$, and polymerized for $24 \mathrm{~h}$. In step 3, after the polymerization, the polymer colloid was dialyzed for 3 days by changing water two times per day to remove to unreacted reactants. The product was freeze-dried for $24 \mathrm{~h}$. In step 4 , the product was eluted with a mixture of methanol/acetic acid $(9: 1, \mathrm{v} / \mathrm{v})$ and water until 4-nitrophenol could not be detected in the eluent by UV-Vis spectroscopy at the wavelength of $317 \mathrm{~nm}$. The product was subsequently washed by water until $\mathrm{pH}$ was 7 . The sample was freeze-dried for $24 \mathrm{~h}$, which yielded CD-MIP. We also studied the effect of drying methods on adsorption capacity. In addition to freeze-drying, the sample was dried under vacuum at different temperatures $\left(25^{\circ} \mathrm{C}, 40^{\circ} \mathrm{C}\right.$ and $60{ }^{\circ} \mathrm{C}$ ) for $24 \mathrm{~h}$. In the present paper, not specifying the drying method in the results means that the freezedried samples were used for the experiments.

As a control, a blank sample was synthesized without adding 4-nitrophenol. This sample is named as celluloseg-DMAEMA-based non-imprinted polymer (CD-NIP).

\section{Effect of $\mathrm{pH}$ on 4-Nitrophenol Adsorption}

The adsorption and desorption of 4-nitrophenol are highly dependent on $\mathrm{pH}$. Therefore, obtaining a complete profile for the effect of $\mathrm{pH}$ variations on adsorption is critical. Investigation of the effect of $\mathrm{pH}$ on the adsorption of 4-nitrophenol was conducted within the $\mathrm{pH}$ range of $3-7$. The procedure is described as follows. CD-MIP (10.0 mg) was mixed with the 4-nitrophenol solution $(25 \mathrm{~mL}, 50 \mathrm{mg} / \mathrm{L})$ at different $\mathrm{pH}$ values ( $\mathrm{pH}$ was adjusted by $0.1 \mathrm{M} \mathrm{NaOH}$ or $0.1 \mathrm{M}$ $\mathrm{HCl}$ ), and the mixture was stirred for $2 \mathrm{~h}$. The residual of 4-nitrophenol in the supernatant was determined by UV-Vis spectroscopy. A predetermined calibration curve was used to calculate the concentration by its absorbance. The same experiments were repeated for CD-NIP as controls. All experiments were repeated 3 times to ensure reproducibility.

\section{Batch Adsorption of 4-nitrophenol}

Batch adsorption of 4-nitrophenol was conducted for both CD-MIP and CD-NIP. The solutions of 4nitrophenol $(25 \mathrm{~mL})$ were mixed with $10 \mathrm{mg}$ CDMIP or CD-NIP in a flask. The solutions were stirred for $2 \mathrm{~h}$ to achieve adsorption equilibrium at different 
temperatures $(298 \mathrm{~K}, 308 \mathrm{~K}$, and $318 \mathrm{~K})$. The concentration of 4-nitrophenol in the residue was determined by UV-Vis spectroscopy. The adsorption capacity was calculated with the following equation,

$Q_{t}=\frac{V\left(C_{0}-C_{e}\right)}{W}$

where $\mathrm{Q}_{\mathrm{t}}(\mathrm{mg} / \mathrm{g})$ is the adsorption capacity, $\mathrm{V}(\mathrm{mL})$ is the volume of the solution, $\mathrm{W}(\mathrm{mg})$ is the amount of CD-MIP or CD-NIP, $\mathrm{C}_{0}$ and $\mathrm{C}_{\mathrm{e}}(\mathrm{mg} / \mathrm{L})$ are the initial concentration of 4-nitrophenol and the 4-nitrophenol concentration once the adsorption reached equilibrium, respectively.

The adsorption isotherm was determined with five initial concentrations of 4-nitrophenol within the concentration range of $5-300 \mathrm{mg} / \mathrm{L}$. The kinetic studies were conducted with the initial concentration of 4-nitrophenol at $50 \mathrm{mg} / \mathrm{L}$. All experiments were repeated three times.

\section{The Selectivity of the Adsorption}

The selectivity of adsorbing 4-nitrophenol was studied by comparing to three other pollutants, 3-nitrophenol, catechol, and hydroquinone. Two experiments were designed. In experiment (1), the adsorption capacity for each pollutant was determined and was compared with the other pollutants. In experiment (2), 4-nitrophenol was mixed with each one of the three other pollutants in binary systems and the competitive adsorption was studied. In experiment (1), the same was applied for all the adsorption experiments regardless of the pollutant, used. The conditions for the experiments include the following: the initial concentration of the pollutants was $50 \mathrm{mg} / \mathrm{L}$, the amount of CD-MIP was $10 \mathrm{mg}$; the equilibrating time was $2 \mathrm{~h}$; the temperature was $298 \mathrm{~K}$. The $\mathrm{pH}$ of all the solutions was 7 . The concentration of each pollutant was determined by UV-Vis spectroscopy at different wavelengths, namely $317 \mathrm{~nm}$ (4-nitrophenol), $273 \mathrm{~nm}$ (3-nitrophenol), at $274 \mathrm{~nm}$ (catechol), and $286 \mathrm{~nm}$ (hydroquinone). Calibration curves were determined for each pollutant to convert absorbance to concentration. In experiment (2), binary systems were used to measure competing adsorption capacity 4-nitrophenol and another pollutant. The initial concentration of each pollutant was $25 \mathrm{mg} / \mathrm{L}$, meaning that the total concentration of the pollutants in the binary systems was $50 \mathrm{mg} / \mathrm{L}$.
Recyclability of CD-MIP

To recycle CD-MIP upon completion of an adsorption experiment, the used CD-MIP was first washed by a mixture of methanol/acetic acid (9:1, v/v), and afterwards washed by water. Thus, the adsorption capacity of the recycled CD-MIP was four further cycles (in total, measurements were performed for measured for five cycles).

\section{Characterization Methods}

The synthesized materials (CD-MIP and CD-NIP) were characterized by various methods. The morphology of the materials was observed by scanning electron microscopy (SEM, LEO-1430VP, Zeiss, Germany) and transmission electron microscopy (TEM, JEM-2100, Hitachi, Japan). Chemical properties were characterized by Fourier transform infrared spectroscopy (FTIR, Vertex 70, Bruker, Germany) using $\mathrm{KBr}$ disk, and the spectra were recorded within the wavenumber range of $500-4000 \mathrm{~cm}^{-1}$. The thermal stability of the materials was evaluated by using a thermal gravimetric analyzer (STA7300, Hitachi, Japan) in a temperature range of $30-600{ }^{\circ} \mathrm{C}$. The rate of temperature increment was $10{ }^{\circ} \mathrm{C} / \mathrm{min}$, and samples were in an $\mathrm{N}_{2}$ atmosphere. The $\mathrm{UV}-\mathrm{V}$ is spectroscopy was conducted on a UV1901PCS instrument (Yoke, Shanghai).

\section{Results and Discussions}

The Experimental Design and the Properties of CD-MIP

The experimental design is depicted in Fig. 1. Cellulose was used as a framework to synthesize CD-MIP. To chemically modify cellulose, it was functionalized first by 2-bromo-2-methylpropionyl bromide. The advantage of using 2-bromo-2-methylpropionyl bromide was that the reaction did not require any catalyst. To generate imprints with a certain type of selectivity, a monomer (DMAEMA) was simply polymerized in the presence of the desired target molecule (4nitrophenol). During the polymerization, the functional groups in DMAEMA oriented towards the counteracting partners in the template, where simultaneously DMAEMA was grafted on cellulose and 


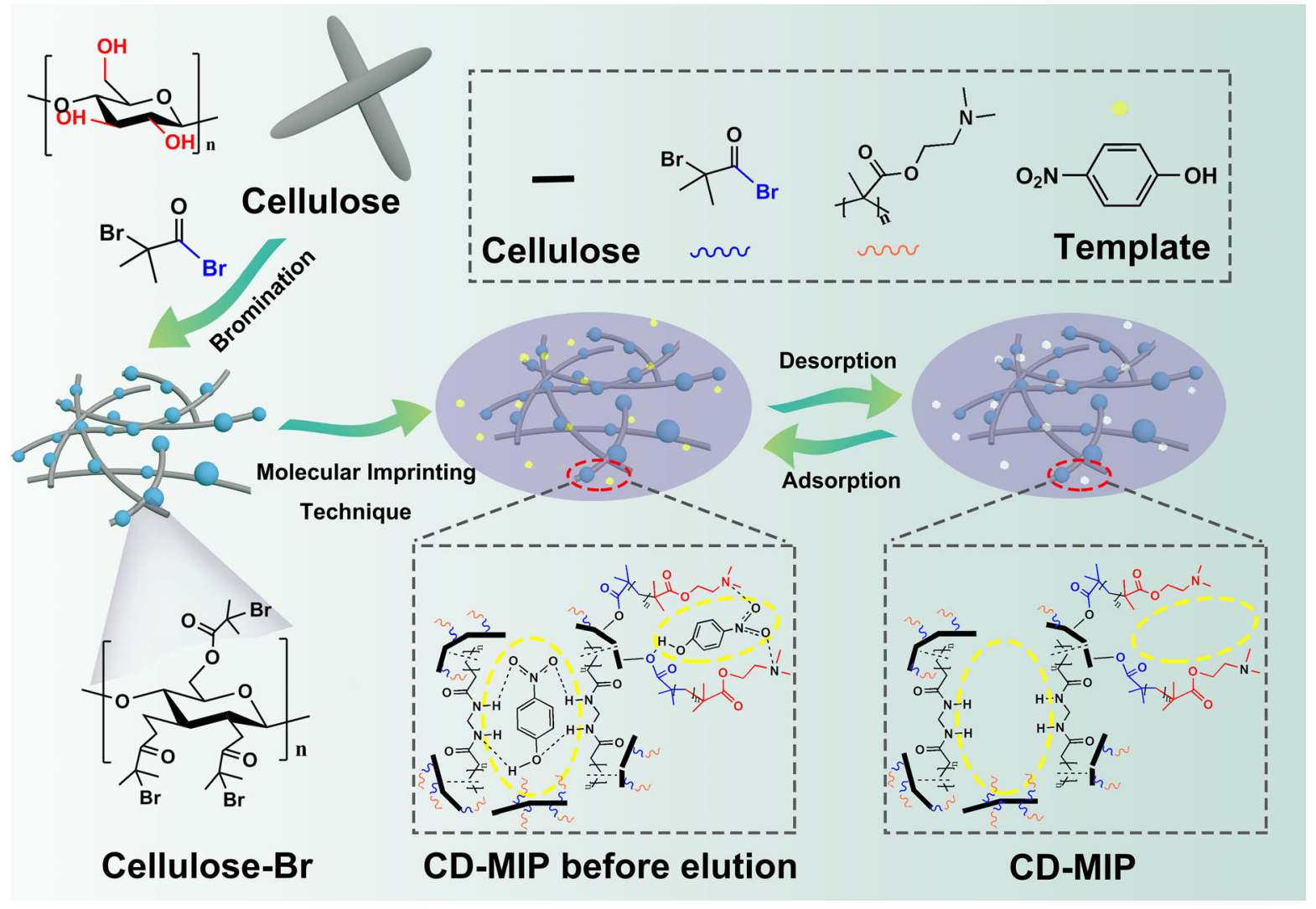

Fig. 1 Schematic illustration of experimental design of synthesizing CD-MIP
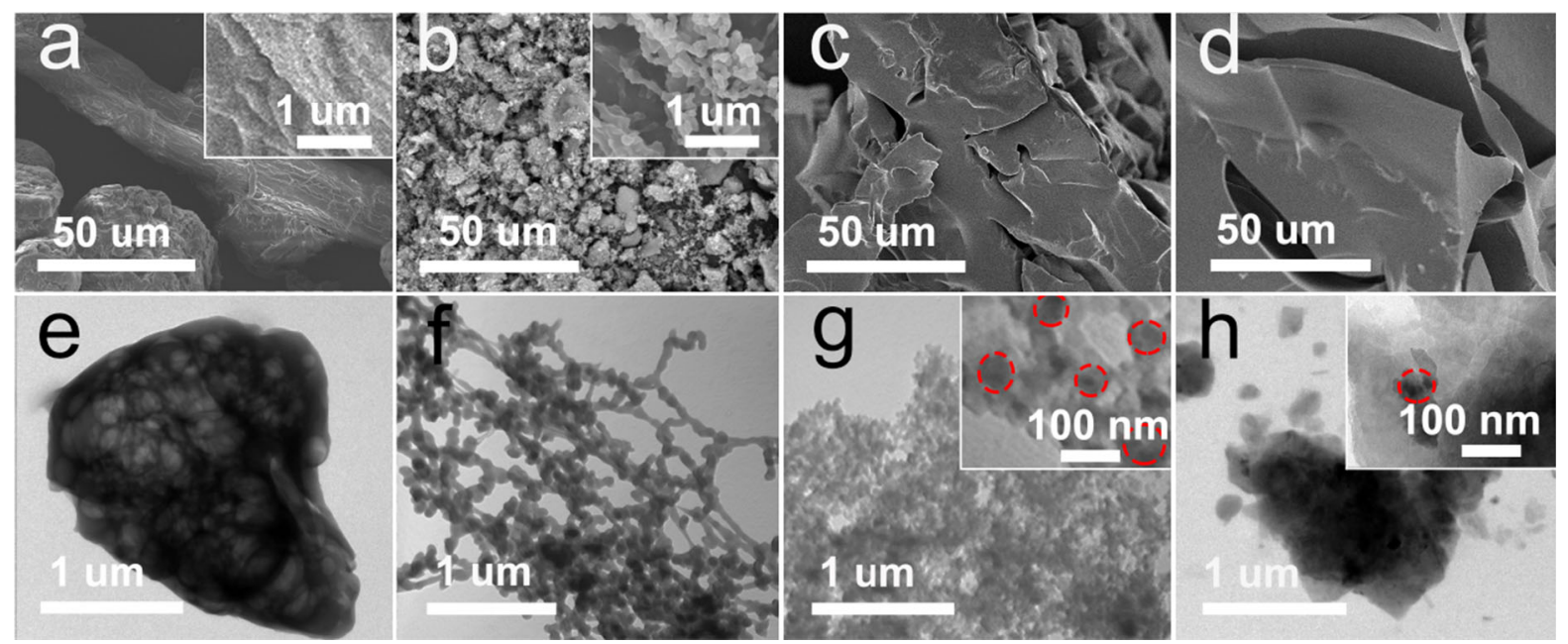

Fig. 2 SEM micrographs of cellulose (a), cellulose-Br (b), CD-MIP (c), CD-NIP (d) and TEM micrographs of cellulose (e), cellulose$\mathrm{Br}(\mathbf{f}), \mathrm{CD}-\mathrm{MIP}(\mathbf{g})$, and CD-NIP (h)

was polymerized, resulting in freezing the orientation of the functional groups. Afterwards, 4-nitrophenol was eluted by using an acidic solution. Therefore, the empty cavities left behind resembled the size and shape of 4-nitrophenol and remembered its surface chemistry. Thus, these cavities would preferentially 

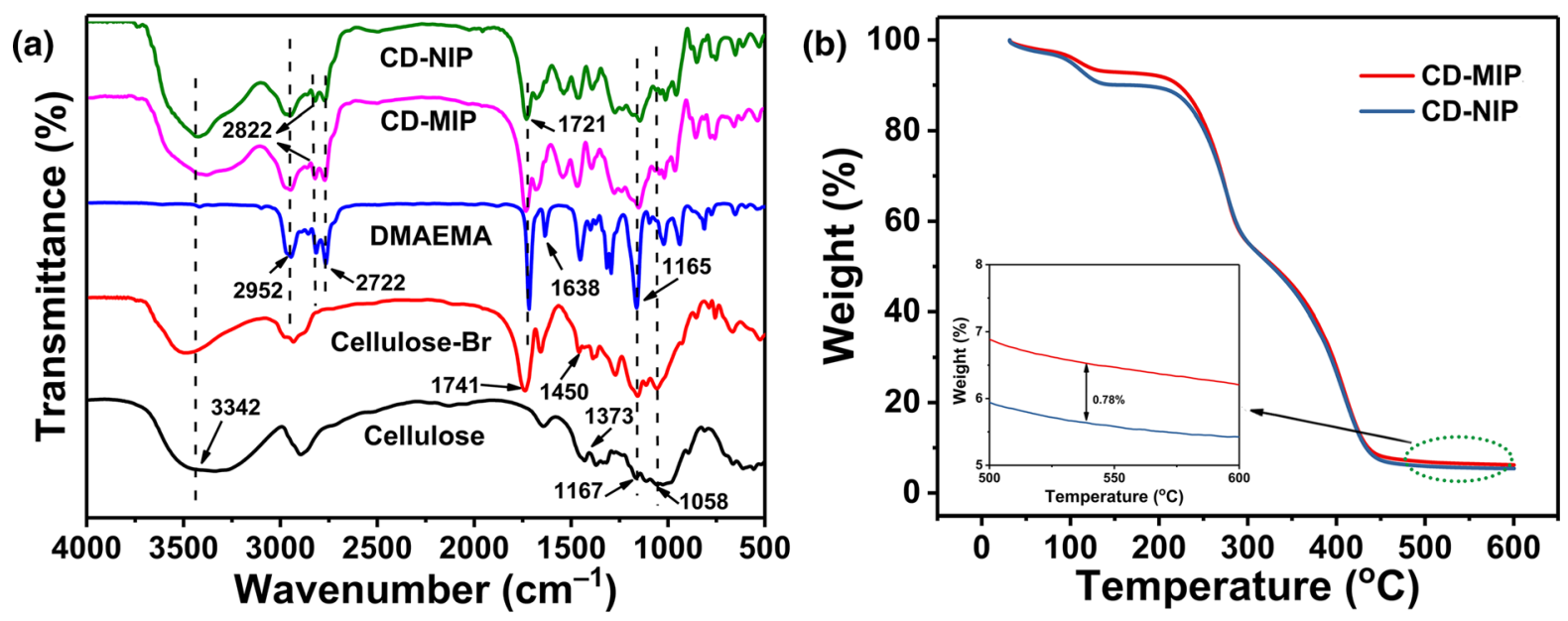

Fig. 3 FTIR spectra of cellulose, cellulose-Br, DMAEMA, CD-MIP, and CD-NIP (a); TGA curves of CD-MIP and CD-NIP (b)

incorporate 4-nitrophenol during the subsequent adsorption experiments.

The morphology of the as-prepared samples in each step (Fig. 1) was first characterized by SEM and TEM (Fig. 2). The morphology of Cellulose was rod-shaped (Fig. 2a), featuring a smooth surface and a porous internal structure (Fig. 2e). This special structure was conducive to an adsorptive material. Dissolution of cellulose in AmimCl could result in a changed morphology. Indeed, after the bromination step, cellulose- $\mathrm{Br}$ had a pearl-necklace-like structure, where small spheres were linked on a string (Fig. 2b, f). The new morphology of cellulose- $\mathrm{Br}$ imparted it better solvent dispersion and dissolution properties compared to cellulose. This "pearl-necklace" structure was conducive to the grafting reaction due to its large surface area. After the grafting, the morphology of the polymer changed again. SEM images revealed minor morphological differences between CD-MIP and CD-NIP, where both appeared as large chunks with smooth surfaces (Fig. 2c, d). Nevertheless, on a smaller length scale (TEM images of Fig. 2g, h), the morphologies of CD-MIP and CD-NIP showed major differences due to the presence of 4-nitrophenol, meaning that while CD-MIP was in a dispersed state the morphology of CD-NIP exhibited an aggregated state, showing large black blocks.

Cellulose-Br, CD-MIP, and CD-NIP were also characterized by FTIR (Fig. 3a). The FTIR spectra of cellulose and DMAEMA were also provided as a reference. Cellulose showed characteristic peaks at $3342 \mathrm{~cm}^{-1}$ (the stretching of $\mathrm{O}-\mathrm{H}$ vibrations),
$1167 \mathrm{~cm}^{-1}$ (stretching vibration of the $\mathrm{C}-\mathrm{O}-\mathrm{C}$ bond) and $1058 \mathrm{~cm}^{-1}$ (anti-stretching vibration of the $\mathrm{C}-\mathrm{O}-$ $\mathrm{C}$ bond) (Kenawy et al. 2018; Mei et al. 2019). For cellulose- $\mathrm{Br}$, we observed additional adsorption at $1741 \mathrm{~cm}^{-1}$ ( $\mathrm{C}=\mathrm{O}$ after acylation), and the $\mathrm{CH}_{3}$ peak corresponding to bromoisobutyl at $1450 \mathrm{~cm}^{-1}$.

For CD-MIP and CD-NIP, the FTIR spectra were almost identical. Cellulose and DMAEMA characteristic peaks were present in both CD-MIP and CD-NIP. The absorption peaks at $2822 \mathrm{~cm}^{-1}$ and $2722 \mathrm{~cm}^{-1}$ were assigned to the stretching vibration of $\mathrm{C}-\mathrm{H}$ in $\mathrm{O}-\mathrm{CH}_{3}$ group; the peak at $1721 \mathrm{~cm}^{-1}$ was assigned to the bending vibration of $\mathrm{C}-\mathrm{C}=\mathrm{O}$; the peak at $1165 \mathrm{~cm}^{-1}$ was the $\mathrm{C}-\mathrm{N}$ characteristic peak (Khakzad et al. 2016); the peak at $1638 \mathrm{~cm}^{-1}$ corresponded to $\mathrm{C}=\mathrm{C}$ stretching vibration. The only difference in the two spectra was the characteristic peak of the $\mathrm{O}-\mathrm{H}$ group above $3000 \mathrm{~cm}^{-1}$, indicating that the status of hydrogen bonding of $\mathrm{OH}$ groups was different in these materials. The FTIR spectra of CD-MIP and CD-NIP confirmed that DMAEMA monomers have been successfully grafted on cellulose.

Thermogravimetric analysis (TGA) was used to evaluate the thermal stability of CD-MIP and CD-NIP. The weight loss was recorded in the temperature range of $30-600{ }^{\circ} \mathrm{C}$ (Fig. 3b). The weight loss for both materials was also remarkably similar, which is expected because the chemical composition of the two materials was identical. There was a deviation in both curves in the range of $100-250{ }^{\circ} \mathrm{C}$, which was due to water loss. The water content in the two materials was different, which was also suggested by 

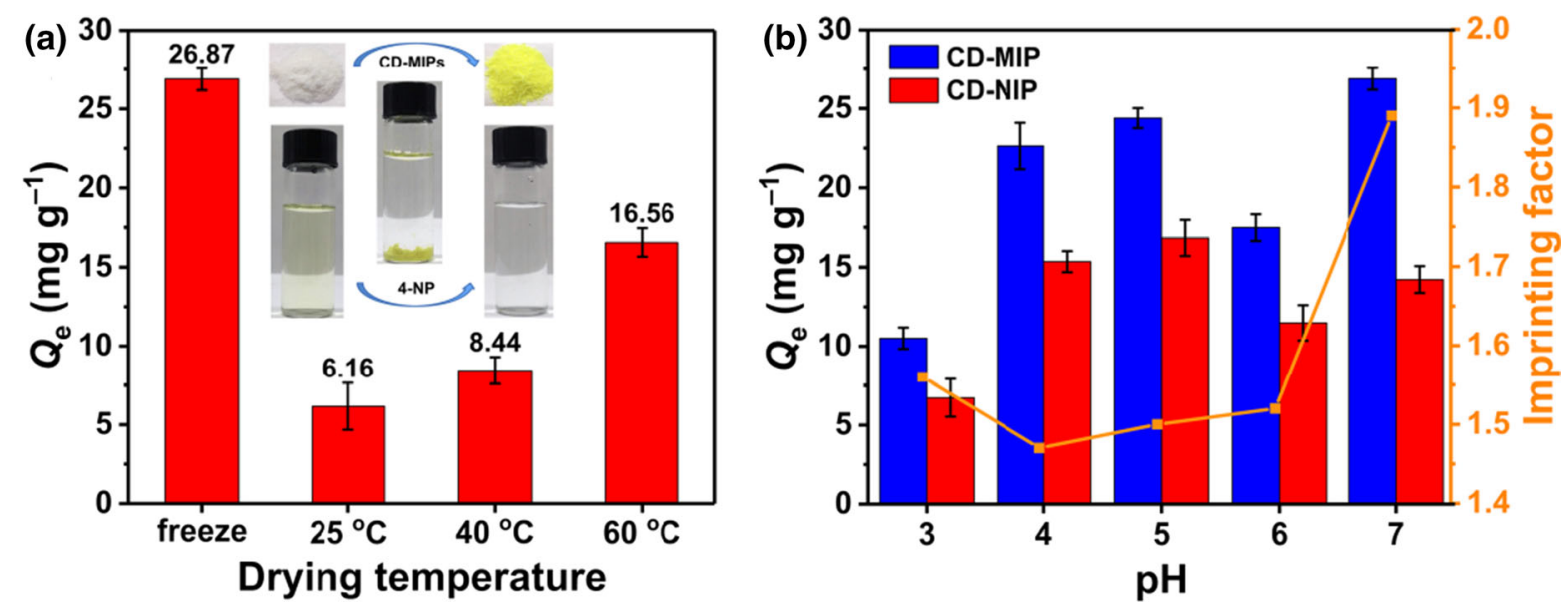

Fig. 4 Effects of drying temperature on the adsorption capacity; (inset) color change of CD-MIP and 4-nitrophenol solution before and after adsorption (a); effect of $\mathrm{pH}$ on the adsorption capacity and imprinting factor (b)

the analysis of the FTIR spectra. CD-MIP had less bound water on its surface compared to CD-NIP, attributed to the configuration of the polymer chain and orientation of functional groups. The weight loss of the polymer framework occurred in $250-500{ }^{\circ} \mathrm{C}$. At $550{ }^{\circ} \mathrm{C}$, the difference in the weight loss in of the two materials was only $0.78 \%$ of the original mass, which indicates that the material synthesis had a good reproducibility ( $\mathrm{Li}$ et al. 2018).

The Effects of Drying Temperature and $\mathrm{pH}$ on Adsorption Capacity

DMAEMA is an interesting monomer, because its tertiary amine functionality shows a strong affinity to 4-nitrophenol in the resulting CD-MIP polymer, (Fig. 4a inserted). The white color of the power of CD-MIP turned into yellow once 4-nitrophenol was adsorbed, and the yellow color of the solution faded. Therefore, this tertiary amine functionality could impart distinct physicochemical properties, e.g., upon changes in temperature and $\mathrm{pH}$. Thus, the effects of the drying temperature and $\mathrm{pH}$ on the adsorption of 4-nitrophenol were investigated. For the samples dried with different methods, the adsorption capacity was measured at room temperature, where the $\mathrm{pH}$ of the solution was 7. A clear difference in adsorption capacity was observed for the CD-MIP (Fig. 4a). The CD-MIP dried at higher temperatures showed better adsorption capacity, whereas the freeze-dried
CD-MIP showed the best adsorption capacity (26.87 mg/g).

The protonation states of 4-nitrophenol and DMAEMA changed with the $\mathrm{pH}$ of the solution, which also affected the adsorption of 4-nitrophenol on CD-MIP and CD-NIP (Fig. 4b). The pKa values of 4-nitrophenol and DMAEMA are 7.15 and $7.0 \sim 7.3$, respectively. Therefore, when the $\mathrm{pH}$ value is below 7 , 4-nitrophenol and DMAEMA are both protonated. The adsorption capacity of CD-MIP decreased with $\mathrm{pH}$, where the highest adsorption capacity $(26.87 \mathrm{mg} /$ g) was observed at $\mathrm{pH}=7$. However, the adsorption capacity of CD-NIP showed a maximum $(17 \mathrm{mg} / \mathrm{g})$ at $\mathrm{pH}=5$, where at $\mathrm{pH}=7$ the adsorption capacity was about $14.23 \mathrm{mg} / \mathrm{g}$.

Imprinting factor (IF) is a measure of the imprinting effect of an adsorbent. The factor is defined as the ratio of the adsorption-capacity of the imprinted adsorbent to the non-imprinted one. The imprinting factor for CD-MIP exhibited the highest value (about 1.89) at $\mathrm{pH}=7$, indicative of a selective adsorption at this $\mathrm{pH}$. Considering the effects of the drying methods and the $\mathrm{pH}$ on the adsorption, we decide to use freezing-dried samples in the studies of adsorption thermodynamics and kinetics. The adsorption process was studied at $\mathrm{pH}=7$, due to its highest imprinting factor value. 

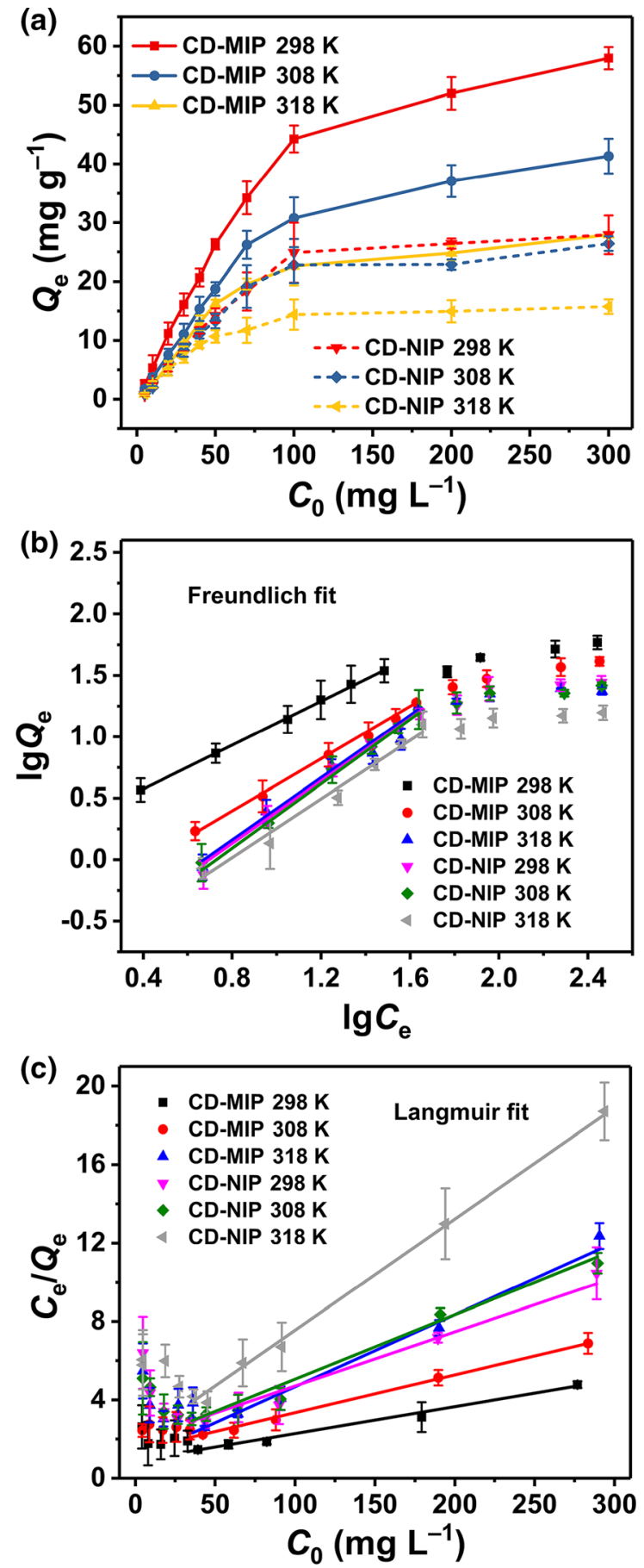

Fig. 5 The adsorption isotherms of 4-nitrophenol on CD-MIP and CD-NIP ( $\mathrm{pH}=7, \mathrm{t}=2 \mathrm{~h})(\mathbf{a})$; Fitting of the Freundlich model to the adsorption data (b); Fitting of the Langmuir model to the adsorption data (c)
Thermodynamics of Adsorption

The thermodynamics of adsorption is important for evaluating the adsorption behavior. The adsorption of 4-nitrophenol was evaluated within the concentration range of 5-300 $\mathrm{mg} / \mathrm{L}$ for CD-MIP and CD-NIP. Three adsorption isotherms were recorded for each adsorbent at $298 \mathrm{~K}, 308 \mathrm{~K}$, and $318 \mathrm{~K}$ (Fig. 5). For both adsorbents, the adsorbed amount decreased with an increase of temperature. All the isotherms exhibited characteristic monolayer adsorption with a fast increase of adsorbed amount at a low initial concentration, which reached saturation at high concentrations (Fig. 5a). For most of the isotherms, the adsorption started to reach saturation once the initial concentration was $100 \mathrm{mg} / \mathrm{L}$.

Since the isotherms represent the typical behavior of monolayer adsorption, we analyzed the data by using the Langmuir model (Zhang et al. 2020):

$\frac{C_{e}}{Q_{e}}=\frac{C_{e}}{\Gamma}+\frac{1}{k_{L} \cdot \Gamma}$

where $\mathrm{C}_{\mathrm{e}}$ is the concentration of adsorbate at equilibrium, $\mathrm{Q}_{\mathrm{e}}$ is the adsorption capacity at equilibrium, $\mathrm{k}_{\mathrm{L}}$ is Langmuir constant, and $\Gamma$ is the adsorbed amount once the adsorption in the monolayer reaches saturation. However, the adsorption data behaved remarkably different at low and high initial concentration (Fig. 5c). Thus, the isotherm at low initial concentrations was treated with the Freundlich model (Zhou et al. 2019):

$\lg Q_{e}=\lg k_{F}+\frac{1}{n} \lg C_{e}$

where $\mathrm{k}_{\mathrm{F}}$ is Freundlich constant and $\mathrm{n}$ is a fitting parameter. In the plot depicting fitting of Freundlich model (Fig. 5b), the data sets also showed two distinctive adsorption modes. The adsorption followed the Freundlich model at low initial concentrations of the adsorbate. In our case, the data with $\mathrm{C}_{0}$ below $50 \mathrm{mg} / \mathrm{L}$ showed good agreement with the model. The extracted fitting parameters ( $\mathrm{n}$ and $\mathrm{k}_{\mathrm{F}}$ ) are presented in Table 1. The Freundlich constant decreased with the temperature and showed much higher for CD-MIP than for CD-NIP. Due to the empirical nature of the model, it was difficult to compare the adsorption behaviors based on $\mathrm{k}_{\mathrm{F}}$. Generally, when one has $0<\frac{1}{n}<1$, the adsorption is deemed favorable. Here, only the adsorption at $298 \mathrm{~K}$ fell into this category. 
Table 1 The fitting parameters for the Freundlich isotherm and the Langmuir isotherm, and the thermodynamic parameters computed based on the Langmuir isotherm

\begin{tabular}{|c|c|c|c|c|c|c|c|c|c|c|}
\hline \multirow[t]{2}{*}{ Absorbents } & \multirow{2}{*}{$\begin{array}{l}\text { Temp. } \\
(\mathrm{K})\end{array}$} & \multicolumn{4}{|c|}{ Freundlich isotherm } & \multicolumn{5}{|c|}{ Langmuir isotherm } \\
\hline & & $1 / \mathrm{n}$ & $\begin{array}{l}\mathrm{k}_{\mathrm{F}}(\mathrm{L} / \\
\mathrm{mg})\end{array}$ & $\mathrm{R}^{2}$ & $\begin{array}{l}\Gamma(\mathrm{mg} / \\
\mathrm{g})\end{array}$ & $\begin{array}{l}\mathrm{k}_{\mathrm{L}}(\mathrm{L} / \\
\mathrm{mg})\end{array}$ & $\mathrm{R}^{2}$ & $\begin{array}{l}\Delta \mathrm{G}^{\circ} \\
(\mathrm{kJ} / \mathrm{mol})\end{array}$ & $\begin{array}{l}\Delta \mathrm{H}^{\circ} \\
(\mathrm{kJ} / \mathrm{mol})\end{array}$ & $\Delta \mathrm{S}^{\circ}(\mathrm{J} / \mathrm{mol} \mathrm{K})$ \\
\hline \multirow[t]{3}{*}{ CD-MIP } & 298 & 0.894 & 1.648 & 0.999 & 72.67 & 0.015 & 0.988 & -21.17 & -23.82 & -8.99 \\
\hline & 308 & 1.067 & 0.348 & 0.998 & 51.87 & 0.014 & 0.991 & -20.82 & & \\
\hline & 318 & 1.269 & 0.140 & 0.980 & 27.17 & 0.037 & 0.981 & -21.05 & & \\
\hline \multirow[t]{3}{*}{ CD-NIP } & 298 & 1.272 & 0.130 & 0.994 & 36.68 & 0.014 & 0.994 & -19.37 & -12.39 & 23.66 \\
\hline & 308 & 1.313 & 0.110 & 0.998 & 30.37 & 0.019 & 0.986 & -19.81 & & \\
\hline & 318 & 1.195 & 0.115 & 0.975 & 17.61 & 0.030 & 0.983 & -19.83 & & \\
\hline
\end{tabular}

If we described the adsorption behavior by the Langmuir isotherm at the low concentration range, the plot of $\mathrm{C}_{\mathrm{e}} \sim \mathrm{C}_{\mathrm{e}} / \mathrm{Q}_{\mathrm{e}}$ often showed a negative slope. Conversely, in the initial concentration range of 50-300 $\mathrm{mg} / \mathrm{L}$, the adsorption exhibited a good agreement with the Langmuir model (Fig. 5c). In Table 1, the results of the fittings and the thermodynamic parameters calculated based on the Langmuir constant $\left(\mathrm{k}_{\mathrm{L}}\right)$ are presented (Man et al. 2015). The adsorption capacity at saturation $(\Gamma)$ showed a clear decrease upon an increase in the temperature for both CD-MIP and CD-NIP. This means that the adsorption was exothermic, which is also reflected in the extracted enthalpy values. The $\Gamma$ value for CD-MIP was twice of that for CD-NIP at $298 \mathrm{~K}$. For the other two temperatures $(308 \mathrm{~K}$ and $318 \mathrm{~K}$ ), the $\Gamma$ values for CD-MIP were around 1.7 times and 1.5 times of those for $\mathrm{CD}$ NIP, respectively. The Langmuir adsorption constant can be understood as the ratio of the rates of adsorption and desorption. For CD-MIP and CD-NIP, the values of the Langmuir constant were similar at all temperatures, indicating that the rates of adsorption and desorption might not change upon temperature variations for both polymers.

Using on the adsorption constant at equilibrium, we computed various thermodynamic parameters (Table 1). The Gibbs free energy showed a small difference for both polymers. Conversely, the enthalpy and entropy changes of adsorption were different for CD-MIP and CD-NIP. Since adsorption was an exothermic process, the enthalpy change of adsorption was negative for both polymers. In the Langmuir model, the enthalpy change of adsorption is a direct measure of the binding strength between the adsorbate and the surface. Thus, the adsorption on CD-MIP generated more heat than the adsorption on CD-NIP, which means that the adsorbate-adsorbent binding strength was higher for CD-MIP than for CD-NIP.

\section{Kinetics of Adsorption}

The kinetics of the adsorption was studied at three temperatures for both CD-MIP and CD-NIP (Fig. 6). The experiments recorded the adsorption data within $2 \mathrm{~h}$ (Fig. 6a). The adsorption occurred very quickly within the first $30 \mathrm{~min}$. Afterward, the adsorption gradually reached saturation within $120 \mathrm{~min}$. The adsorption capacity at $120 \mathrm{~min}$ was less than $1 \mathrm{mg} / \mathrm{g}$ higher than its value at $90 \mathrm{~min}$, indicating that the adsorption had reached equilibrium around $120 \mathrm{~min}$. The temperature-dependent nature of the adsorption was also clearly observed in Fig. 6a, where the adsorption capacity exhibited a much higher value at a lower temperature.

The kinetics of adsorption was analyzed by pseudofirst-order (Eq. 4) and pseudo-second-order models (Eq. 5) (Du et al. 2018).

$\ln \left(Q_{e}-Q_{t}\right)=\ln Q_{e}-k_{1} t$

$\frac{t}{Q_{t}}=\frac{1}{k_{2} Q_{e}^{2}}+\frac{1}{Q_{e}}$

where $\mathrm{Q}_{\mathrm{t}}(\mathrm{mg} / \mathrm{g})$ is the adsorption capacity of CD-MIP and CD-NIP at time $\mathrm{t}$, and $\mathrm{k}_{1}\left(\mathrm{~min}^{-1}\right)$ is the rate constant of adsorption for the pseudo-first-order adsorption. In the pseudo-first-order model, the values of $\ln \left(Q_{e}-Q_{t}\right)$ linearly decreased with time (Fig. 6b). For pseudo-second-order adsorption model, the values 

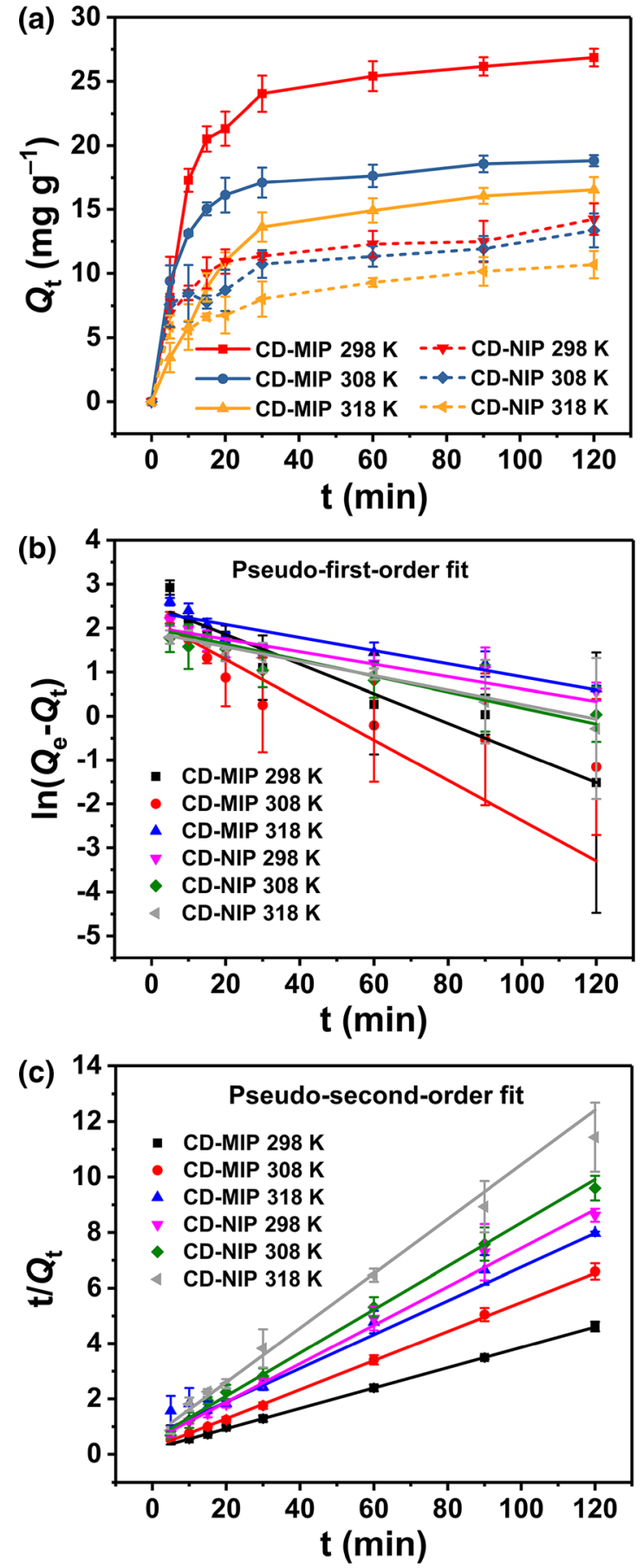

Fig. 6 The kinetics of the adsorption: the adsorption capacity as a function of time for CD-MIP and CD-NIP at three different temperatures $\left(\mathrm{pH}=7, \mathrm{C}_{0}=50 \mathrm{mg} / \mathrm{g}\right)(\mathbf{a})$; the fitting of the pseudo-first-order kinetic model to the data (b); the fitting of the pseudo-second-order kinetic model to the data (c) of $t / Q_{t}$ linearly increased with time (Fig. 6c), where $k_{2}$ ( $\mathrm{g} / \mathrm{mg} \mathrm{min}$ ) was the rate constant of the adsorption for pseudo-second-order kinetic model. The fitting results for the kinetic models are summarized in Table 2. The results were indicative of a good fitting for both models and the theoretical values of Qe obtained by the pseudo-second-order model matched well with the $\mathrm{Q}_{\max }$ experimental values (i.e., the maximum adsorption capacity in the experimental data set). At $298 \mathrm{~K}$, the Qe of CD-MIP was $26.87 \mathrm{mg} / \mathrm{g}$, significantly higher than that of CD-NIP $(14.23 \mathrm{mg} / \mathrm{g})$, indicating that the active sites on CD-MIP had a better affinity to 4-nitrophenol and the rate-controlling step belonged to the chemical adsorption, in which, the chemical bonding force between the adsorbate and the adsorbent played a role.

\section{Selective Adsorption}

Selective adsorption is an important criterion for evaluating MIPs. For this purpose, we chose four chosen phenols, 4-nitrophenol, 3-nitrophenol, catechol, and hydroquinone. The selective adsorption was conducted with two experiments. In experiment (1), comparisons were made between the adsorption capacity for individual phenol (Fig. 7a). In experiment (2), selective adsorption in a binary mixture of the phenols (Fig. 7b) was examined.

In the single-component experiment, CD-MIP showed the highest adsorption capacity for 4-nitrophenol $(26.87 \mathrm{mg} / \mathrm{g})$, and the lowest for 3-nitrophenol $(2.63 \mathrm{mg} / \mathrm{g})$. Another interesting observation is that the difference between the adsorption capacity for CD-MIP and CD-NIP is much higher for 4-nitrophenol than for of the phenols. For example, the adsorption capacity for 4-nitrophenol was $26.87 \mathrm{mg} / \mathrm{g}$ for CD-MIP and $14.23 \mathrm{mg} / \mathrm{g}$ for CD-NIP, which gives a value of $12.64 \mathrm{mg} / \mathrm{g}$ for the difference between the adsorption capacities of the two polymers. Interestingly, for 3-nitrophenol this value was much lower (0.07 mg/g).

This selectivity of adsorption can be quantitatively evaluated by the distribution coefficient $\left(\mathrm{K}_{\mathrm{d}}\right)$, which is defined as (Cao et al. 2014)

$K_{d}=\frac{Q_{e}}{C_{e}}$ 
Table 2 Kinetic constants for the Pseudo-first order and the Pseudo-second-order models

\begin{tabular}{|c|c|c|c|c|c|c|c|}
\hline \multirow[t]{2}{*}{ Absorbents } & \multirow[t]{2}{*}{ Temp. (K) } & \multicolumn{3}{|c|}{ Pseudo first order } & \multicolumn{3}{|c|}{ Pseudo second order } \\
\hline & & $\mathrm{Q}_{\mathrm{e}}(\mathrm{mg} / \mathrm{g})$ & $\mathrm{k}_{1}(1 / \mathrm{min})$ & $\mathrm{R}^{2}$ & $\mathrm{Q}_{\mathrm{e}}(\mathrm{mg} / \mathrm{g})$ & $\mathrm{k}_{2} \times 10^{-2}(\mathrm{~g} / \mathrm{mg} \min )$ & $\mathrm{R}^{2}$ \\
\hline \multirow[t]{3}{*}{ CD-MIP } & 298 & 12.564 & 0.034 & 0.684 & 27.218 & 0.007 & 0.999 \\
\hline & 308 & 9.085 & 0.046 & 0.688 & 19.048 & 0.012 & 0.998 \\
\hline & 318 & 10.829 & 0.015 & 0.911 & 16.415 & 0.006 & 0.998 \\
\hline \multirow[t]{3}{*}{ CD-NIP } & 298 & 7.596 & 0.014 & 0.827 & 14.407 & 0.010 & 0.997 \\
\hline & 308 & 7.391 & 0.018 & 0.859 & 12.804 & 0.011 & 0.985 \\
\hline & 318 & 6.715 & 0.016 & 0.916 & 10.192 & 0.015 & 0.981 \\
\hline
\end{tabular}

(a)

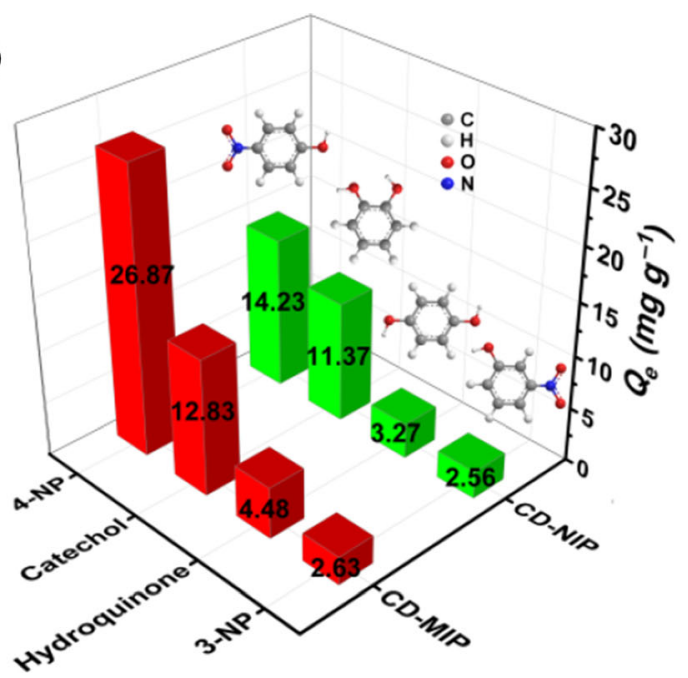

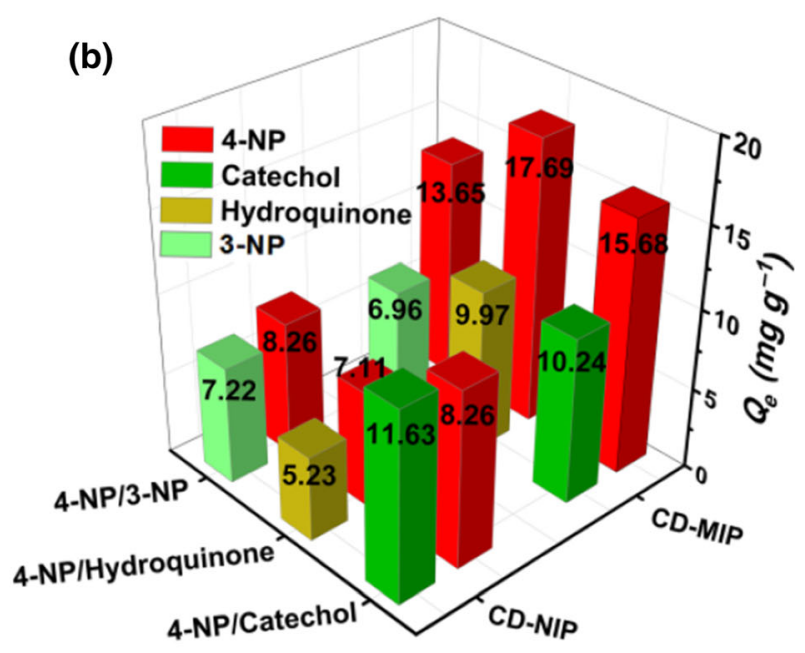

Fig. 7 The adsorption capacity of CD-MIP and CD-NIP for four different phenols in single-component solutions (a); the adsorption capacity of CD-MIP and CD-NIP in binary solutions of 4-nitrophenol and another phenol (b)
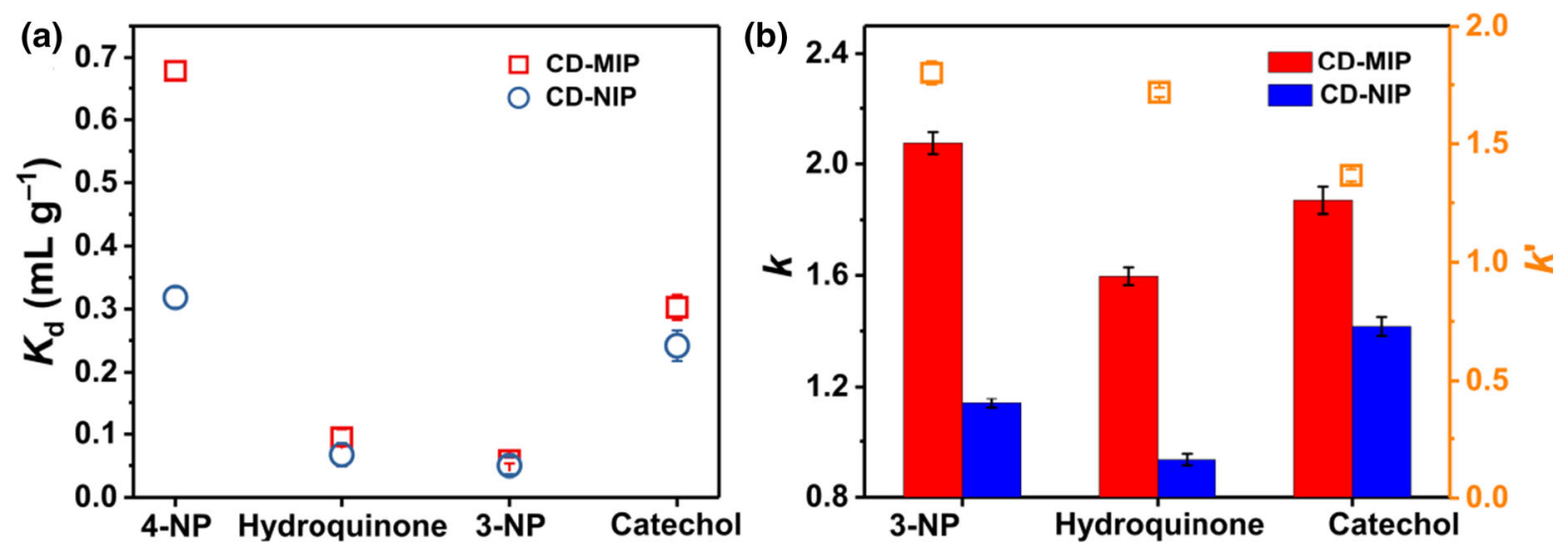

Fig. 8 In CD-MIP and CD-NIP materials, $\mathrm{K}_{\mathrm{d}}$ values for 4-nitrophenol, 3-nitrophenol, catechol and hydroquinone $(\mathrm{pH}=7, \mathrm{~T}=298 \mathrm{~K}$, $\left.\mathrm{C}_{0}=50 \mathrm{mg} / \mathrm{g}\right)(\mathbf{a})$, and $\mathrm{k}$ and $\mathrm{k}$ ' values for 3-nitrophenol, catechol and hydroquinone (b) 
where $\mathrm{Q}_{\mathrm{e}}$ and $\mathrm{C}_{\mathrm{e}}$ are the equilibrium concentration of the adsorbate in the adsorbent and the supernatant, respectively. For 4-nitrophenol, the value of $\mathrm{K}_{\mathrm{d}}$ of $\mathrm{CD}$ MIP $(0.661 \mathrm{~mL} / \mathrm{g})$ was much higher than those of the other phenols (Fig. 8a). For 4-nitrophenol, the values of $\mathrm{K}_{\mathrm{d}}$ for CD-MIP and CD-NIP were also profoundly different. Conversely, for the other phenols, the difference of $\mathrm{K}_{\mathrm{d}}$ for CD-MIP and CD-NIP was exceedingly small. The difference in $K_{d}$ values for CD-MIP and CD-NIP quantitatively described the selectivity of the adsorption, thus identifying a particularly good selectivity for 4-nitrophenol by CD-MIP.

In experiments (2) conducted for determining the selectivity of adsorption, the difference in the adsorption capacities for a binary mixture of 4-nitrophenol with another phenol was investigated. The results also showed excellent selectivity of CD-MIP for 4-nitrophenol (Fig. 7b). With CD-MIP, the adsorption capacity for 4-nitrophenol was much higher than that for 3-nitrophenol, hydroquinone, and catechol. Conversely, CD-NIP did not show a selectivity in the adsorption of 4-nitrophenol over the other phenols. In the binary mixture of 4-nitrophenol/catechol, the adsorption capacity of CD-NIP for 4-nitrophenol was even lower than that for catechol. In the binary systems, the selective adsorption can be quantitatively evaluated by selectivity coefficient (k) (Zhou et al. 2020), which can be defined as:

$k=\frac{K_{d}(4-\text { nitrophenol })}{K_{d}(\text { other phenol })}$

where the $K_{d}$ values of 4-nitrophenol and other phenols can be computed using Eq. 6. The results showed a clear difference in the selective adsorption for MIP in comparison to NIP (Fig. 8b). For MIPs, the $\mathrm{k}$ values were around 2 for all of the other three phenols, which meant that the affinity of MIPs for 4nitrophenols was twice of those for the other phenols. However, for NIPs, the values of $\mathrm{k}$ were about 1 for 3nitrophenol and hydroquinone, indicating that there was no difference in the affinity of NIPs for 4nitrophenol and these two phenols. The $\mathrm{k}$ value for 4nitrophenol/catechol mixture for NIP was about 1.5, which meant that even for NIP the adsorption was slightly selective, and the selectivity for the 4-nitrophenol/catechol mixture observed in MIP could not be solely attributed to the imprinted effect. The adsorption of 4-nitrophenol might be preferable over the adsorption of catechol due to the chemical composition of the material. This effect could be quantitatively evaluated by the relative selectivity coefficient $\mathrm{k}^{\prime}$, which can be defined as (Zheng et al. 2019):

$k^{\prime}=\frac{k_{M I P}}{k_{N I P}}$

where $\mathrm{k}_{\mathrm{MIP}}$ and $\mathrm{k}_{\mathrm{NIP}}$ are computed using Eq. 7. The values of $\mathrm{k}^{\prime}$ for 3-nitrophenol and hydroquinone are much higher than its value for catechol, indicating that the selectivity of adsorption for these two phenols was mostly due to the imprinted effect. As explained above, the selectivity of the adsorption between 4-nitrophenol and catechol was not only because of the imprinted effect.

To objectively evaluate the adsorption of 4-nitrophenol, we here compared the adsorption capacity in our study with those of other recent publications to give an overview on the progress of developing novel adsorbents for 4-nitrophenol. In Table 3, we summarized 20 studies with diverse types of the adsorbent for 4-nitrophenol, including polymer compositions and carbon materials. The value of $\mathrm{Q}_{\max } / \mathrm{C}_{0}$ was used to evaluate the adsorption capacity in different studies. In the category of selective adsorption, CD-MIP exhibited very high adsorption capacity within a relatively short time. The adsorption capacity was also relatively high among the non-selective adsorbents. However, pure graphene and vermiculites exhibited the best adsorption capacity, which was due to the large surface areas of these materials.

\section{Desorption and Recycling of CD-MIP}

For good adsorbents, the reuse of the material is a crucial factor. Here, the recyclability of CD-MIP was evaluated by $Q_{e}$ and adsorptive efficiency in five cycles. As described in Method, the used CD-MIP was eluted with an acidic solution to remove 4-nitrophenol, and the adsorption was repeated by using the regenerated CD-MIP. $\mathrm{Q}_{\mathrm{e}}$ and adsorptive efficiency were showing in Fig. 9. There was a slight decrease in both values. After 5 cycles, the adsorption capacity was reduced to $24.25 \mathrm{mg} / \mathrm{g}$, and over $90 \%$ of its original adsorptive efficiency was retained. The loss of the adsorptive capacity could be because the template embedded in the adsorbent was not eluted, or the loss of a part of imprinted cavities in the polymer matrix 
Table 3 Comparison of the adsorption capacity CD-MIP with some previously reported adsorbents for 4-nitrophenol

\begin{tabular}{lllll}
\hline Adsorbent & $\mathrm{C}_{0}$ & $\begin{array}{l}\text { Experimental conditions } \\
(\mathrm{mg} /\end{array}$ & $\begin{array}{l}\mathrm{Q}_{\max } \\
(\mathrm{pH}, \mathrm{T}, \mathrm{time})\end{array}$ & $\begin{array}{l}\text { Adsorption } \\
\text { efficiency }\end{array}$ \\
\hline
\end{tabular}

Selective adsorbents

CD-MIP (This work)

MGR@MIPs (Luo et al. 2016)

GO/PNIPAm (Tian et al. 2018)

$\begin{array}{lllll}50 & 7,298 \mathrm{~K}, 2 \mathrm{~h} & 26.87 & 90.2 \% & 0.537 \\ 600 & -,-, 5 \mathrm{~min} & 145 & 95.3 \% & 0.242 \\ 50 & 3,298 \mathrm{~K}, 12 \mathrm{~h} & 19.78 & 85 \% & 0.396 \\ 1252 & 5,298 \mathrm{~K}, 1.5 \mathrm{~h} & 129.1 & 98.5 \% & 0.103 \\ 60 & 6,218 \mathrm{~K}, 12 \mathrm{~h} & 22.11 & 90.2 \% & 0.369 \\ 50 & 3,298 \mathrm{~K}, 10 \mathrm{~h} & 25.9 & - & 0.518 \\ 50 & 3,298 \mathrm{~K}, 2 \mathrm{~h} & 27.6 & - & 0.552\end{array}$

surface-immobilized 4-nitrophenol-MMIP (Mehdinia et al. 2014) AAPTS surface-modified potassium tetratitanate whisker-
MIP (Guan et al. 2011)

Methacryloylantipyrine/4-nitrophenol-MIPs (Say et al. 2005)

Methacryloylantipyrine/4-nitrophenol/MMA-MIPs beads 50

(Say et al. 2005)

Non-selective adsorbents

Pure graphene (Ismail 2015)

Activated jute stick char (Ahmaruzzaman and Gayatri 2010)

PS- $\mathrm{CH}_{2}-\left[\mathrm{C}_{2} \mathrm{NH}_{2} \mathrm{MIm}\right][\mathrm{Br}]$ (Cheng et al. 2019)

aminopropyl-modified MCM-48 (Gu et al. 2018)

PVA (Narkkun et al. 2019)

PVA-L-arginine2 membrane (Narkkun et al. 2019)

PVA- L-arginine3 membrane (Narkkun et al. 2019)

$\begin{array}{lllll}11.1 & 6,298 \mathrm{~K}, 5 \mathrm{~min} & 15.5 & - & 1.396 \\ 1000 & 9.0,298 \mathrm{~K}, 4 \mathrm{~h} & 39.38 & - & 0.039 \\ & & & & \\ 10,000 & -, 293 \mathrm{~K}, 30 \mathrm{~min} & 1269.8 & 97.5 \% & 0.127 \\ 50 & 6.5,293.15 \mathrm{~K}, 45 \mathrm{~min} & 30.14 & 80 \% & 0.603 \\ 100 & -, 303 \mathrm{~K}, 1 \mathrm{~h} & 2.12 & - & 0.021 \\ 100 & -, 303 \mathrm{~K}, 30 \mathrm{~min} & 3.55 & - & 0.036 \\ 100 & -, 303 \mathrm{~K}, 8 \mathrm{~min} & 6.78 & - & 0.068 \\ 50 & -, 298 \mathrm{~K}, 16 \mathrm{~h} & 11.23 & - & 0.225 \\ & & & & 0.968 \\ 50 & 7,298 \mathrm{~K}, 5 \mathrm{~h} & 48.4 & - & 0.190 \\ 100 & 6,308 \mathrm{~K}, 24 \mathrm{~h} & 19 & - & 1.065 \\ 100 & 6,298 \mathrm{~K}, 20 \mathrm{~min} & 106.5 & - & 0.010 \\ & & & & 0.512 \\ 4000 & 8.5,303 \mathrm{~K}, 5 \mathrm{~s} & 41.5 & 75 \% & - \\ 400 & 7,303 \mathrm{~K}, 180 \mathrm{~min} & 204.8 & - & \end{array}$

phthalimidomethyl polysulfone/polysulfone blend affinity membrane (Sun and Wang 2011)

AgTz-1 (Miao et al. 2020)

Cr- terephthalic acid (Chen et al. 2017)

bis(dodecyldimethylammonio)-2-butane dibromide/ organo-vermiculites (Yu et al. 2018)

Silica gel-grafted $\beta$-cyclodextrin (Shen et al. 2015)

Microalgal biochars (Zheng et al. 2017)

during the repeated adsorption-desorption process. The FTIR spectra (Fig. 9b) were used to trace 4-nitrophenol during the process of the elusion and re-adsorption. The characteristic absorptions of the $\mathrm{NO}_{2}$ group in 4-nitrophenol at $1344 \mathrm{~cm}^{-1}$ and $1589 \mathrm{~cm}^{-1}$ were observed after the adsorption. However, there was no notable change in the spectra of the original CD-MIP and the CD-MIP after the elution. In these two spectra, no 4-nitrophenol could be detected, indicating that the adsorbed template was removed to a level below the detective limit of FTIR. Besides, these two spectra were also identical indicating that the internal structure of CD-MIP remained intact after elusion.

\section{Conclusion}

In this study, we aimed at developing an efficient adsorbent for 4-nitrophenol to be used in water treatment. By using molecular imprinting technology (MIT), we employed cellulose as a framework material, onto which, 4-nitrophenol was imprinted by the help of DMAEMA. The cellulose-based MIP exhibited excellent adsorption capacity and selectivity. The 

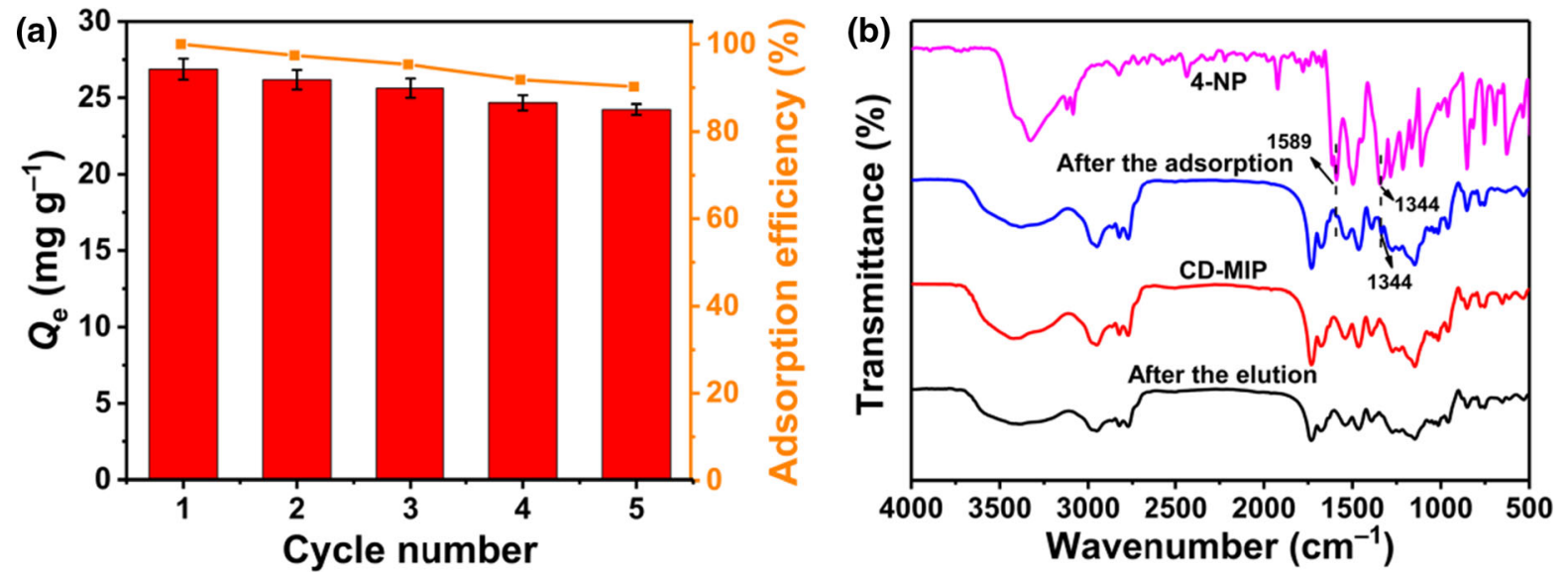

Fig. 9 The adsorbed amount and adsorptive efficiency change with the cycle number (a); FTIR spectra of CD-MIP before the adsorption, after the adsorption of 4-nitrophenol, and after elution (b)

maximum adsorption capacity reached $26.87 \mathrm{mg} / \mathrm{g}$ with an imprinting factor of 1.89 . The selectivity coefficients for the selective adsorption of 4-nitrophenol in binary solutions were 11.69 (4-nitrophenol + 3-nitrophenol),

(4nitrophenol + hydroquinone), and 2.24 (4-nitrophenol + catechol). Moreover, CD-MIP demonstrated excellent stability and recyclability after 5 cycles of consecutive adsorption-desorption.

Acknowledgments We thank financial supports from the Thousand Talent Program, the National Natural Science Foundation of China (NO.21868036), and the Project of Xinjiang Education Office (NO. XJEDU20171004). We also thank the laboratory analysis platform at the Ministry Key Laboratory of Oil \& Gas Fine Chemicals. Language editing by SPEEdS (Stylus Professional English Editing Services) is acknowledged.

Funding Open access funding provided by University of Bergen (incl Haukeland University Hospital).

\section{Declarations}

Conflict of interest The authors declare that they have no conflict of interest.

Ethical approval This research does not contain any studies with humans or animals as subjects.

Informed consent Informed consent was obtained from all of the individual participants included in the study.

Open Access This article is licensed under a Creative Commons Attribution 4.0 International License, which permits use, sharing, adaptation, distribution and reproduction in any medium or format, as long as you give appropriate credit to the original author(s) and the source, provide a link to the Creative Commons licence, and indicate if changes were made. The images or other third party material in this article are included in the article's Creative Commons licence, unless indicated otherwise in a credit line to the material. If material is not included in the article's Creative Commons licence and your intended use is not permitted by statutory regulation or exceeds the permitted use, you will need to obtain permission directly from the copyright holder. To view a copy of this licence, visit http://creativecommons.org/licenses/by/4.0/.

\section{References}

Ahmaruzzaman M, Gayatri SL (2010) Batch adsorption of 4-nitrophenol by acid activated jute stick char: equilibrium, kinetic and thermodynamic studies. Chem Eng J 158:173-180. https://doi.org/10.1016/j.cej.2009.12.027

Alhokbany N, Ahama T, Ruksana NM, Alshehri SM (2019) AgNPs embedded N-doped highly porous carbon derived from chitosan based hydrogel as catalysts for the reduction of 4-nitrophenol. Compos B Eng 173:106950. https://doi. org/10.1016/j.compositesb.2019.106950

Bahrami S, Yaftian MR, Najvak P, Dolatyari L, Shayani-Jam H, Kolev SD (2020) PVDF-HFP based polymer inclusion membranes containing Cyphos ${ }^{\circledR}$ IL 101 and Aliquat ${ }^{\circledR} 336$ for the removal of $\mathrm{Cr}(\mathrm{VI})$ from sulfate solutions. Sep Purif Technol 250:117251. https://doi.org/10.1016/j.seppur. 2020.117251

Belhouchet N, Hamdi B, Chenchouni H, Bessekhouad Y (2019) Photocatalytic degradation of tetracycline antibiotic using new calcite/titania nanocomposites. J Photochem Photobiol A 372:196-205. https://doi.org/10.1016/j.jphotochem. 2018.12.016

Cantarella M, Carroccio SC, Dattilo S, Avolio R, Castaldo R, Puglisi C, Privitera V (2019) Molecularly imprinted polymer for selective adsorption of diclofenac from contaminated water. Chem Eng J 367:180-188. https://doi.org/ 10.1016/j.cej.2019.02.146 
Cao Y, Liu L, Xu W, Wu X, Huang W (2014) Surface molecularly imprinted polymer prepared by reverse atom transfer radical polymerization for selective adsorption indole. J Appl Polym Sci. https://doi.org/10.1002/app.40473

Cao J, Wang Z, Yang X, Tu J, Wu R, Wang W (2018) Green synthesis of amphipathic graphene aerogel constructed by using the framework of polymer-surfactant complex for water remediation. Appl Surf Sci 444:399-406. https://doi. org/10.1016/j.apsusc.2018.02.282

Cegłowski M, Kurczewska J, Ruszkowski P, Schroeder G (2019) Application of paclitaxel-imprinted microparticles obtained using two different cross-linkers for prolonged drug delivery. Eur Polym 118:328-336. https://doi.org/10. 1016/j.eurpolymj.2019.06.010

Chen J, Sun X, Lin L, Dong X, He Y (2017) Adsorption removal of o-nitrophenol and p-nitrophenol from wastewater by metal-organic framework Cr-BDC. Chin. J Chem Eng 25:775-781. https://doi.org/10.1016/j.cjche.2016.10.014

Cheng M, Jiang J, Wang J, Fan J (2019) Highly salt resistant polymer supported ionic liquid adsorbent for ultrahigh capacity removal of p-nitrophenol from water. ACS Sustain Chem Eng 7:8195-8205. https://doi.org/10.1021/ acssuschemeng.8b06198

Cui Z et al (2020) Novel magnetic fluorescence probe based on carbon quantum dots-doped molecularly imprinted polymer for AHLs signaling molecules sensing in fish juice and milk. Food Chem 328:127063. https://doi.org/10.1016/j. foodchem.2020.127063

Du K, Li S, Zhao L, Qiao L, Ai H, Liu X (2018) One-step growth of porous cellulose beads directly on bamboo fibers via oxidation-derived method in aqueous phase and their potential for heavy metal ions adsorption. ACS Sustain Chem Eng 6:17068-17075. https://doi.org/10.1021/ acssuschemeng. 8 b04433

Gericke M, Trygg J, Fardim P (2013) Functional cellulose beads: preparation, characterization, and applications. Chem Rev 113:4812-4836. https://doi.org/10.1021/ cr300242j

Gu X, Kang H, Li H, Liu X, Dong F, Fu M, Chen J (2018) Adsorption removal of various nitrophenols in aqueous solution by aminopropyl-modified mesoporous MCM-48. J Chem Eng Data 63:3606-3614. https://doi.org/10.1021/ acs.jced.8b00477

Guan W, Pan J, Wang X, Hu W, Xu L, Zou X, Li C (2011) Selective recognition of 4-nitrophenol from aqueous solution by molecularly imprinted polymers with functionalized tetratitanate whisker composites as support. J Sep Sci 34:1244-1252. https://doi.org/10.1002/jssc. 201100032

Gulati A, Malik J, Mandeep KR (2020) Peanut shell biotemplate to fabricate porous magnetic $\mathrm{Co}_{3} \mathrm{O}_{4}$ coral reef and its catalytic properties for $\mathrm{p}$-nitrophenol reduction and oxidative dye degradation. Colloid Surface A 604:125328. https:// doi.org/10.1016/j.colsurfa.2020.125328

Ismail AI (2015) Thermodynamic and kinetic properties of the adsorption of 4-nitrophenol on graphene from aqueous solution. Can J Chem 93:1083-1087. https://doi.org/10. 1139/cjc-2014-0450

Jiang L, Liu H, Li M, Xing Y, Ren X (2016) Surface molecular imprinting on CdTe quantum dots for fluorescence sensing of 4-nitrophenol. Anal Methods 8:2226-2232. https://doi. org/10.1039/C5AY03160E

Kenawy I, Ismail M, Hafez M, Hashem M (2018) Synthesis and characterization of novel ion-imprinted guanyl-modified cellulose for selective extraction of copper ions from geological and municipality sample. Int J Biol Macromol 115:625-634. https://doi.org/10.1016/j.jtice.2018.02.019

Khakzad F, Mahdavian AR, Salehi-Mobarakeh H, Shirin-Abadi AR, Cunningham M (2016) Redispersible PMMA latex nanoparticles containing spiropyran with photo-, $\mathrm{pH}$-and $\mathrm{CO}_{2}$-responsivity. Polymer 101:274-283. https://doi.org/ 10.1016/j.polymer.2016.08.073

Koyuncu H, Yıldız N, Salgın U, Köroğlu F, Çalımlı A (2011) Adsorption of o-, m-and p-nitrophenols onto organically modified bentonites. J Hazard Mater 185:1332-1339. https://doi.org/10.1016/j.jhazmat.2010.10.050

Kumari S, Chauhan GS (2014) New cellulose-lysine Schiffbase-based sensor-adsorbent for mercury ions. ACS Appl Mater Interfaces 6:5908-5917. https://doi.org/10.1021/ am500820n

Li Y, Xiao H, Pan Y, Wang L (2018) Novel composite adsorbent consisting of dissolved cellulose fiber/microfibrillated cellulose for dye removal from aqueous solution. ACS Sustain Chem Eng 6:6994-7002. https://doi.org/10.1021/ acssuschemeng.8b00829

Li L et al (2020) Molecularly imprinted carbon nanosheets supported $\mathrm{TiO}_{2}$ : strong selectivity and synergic adsorptionphotocatalysis for antibiotics removal. J Hazard Mater 383:121211. https://doi.org/10.1016/j.jhazmat.2019. 121211

Liang W, Lu Y, Li N, Li H, Zhu F (2020) Microwave-assisted synthesis of magnetic surface molecular imprinted polymer for adsorption and solid phase extraction of 4-nitrophenol in wastewater. Microchem J 159:105316. https:// doi.org/10.1016/j.microc.2020.105316

Liu L, Gao ZY, Su XP, Chen X, Jiang L, Yao JM (2015) Adsorption removal of dyes from single and binary solutions using a cellulose-based bioadsorbent. ACS Sustain Chemi Eng 3:432-442. https://doi.org/10.1021/ sc500848m

Lu Q et al (2020) Using ultrasonic treated sludge to accelerate pyridine and p-nitrophenol biodegradation. Int Biodeter Biodegr 153:105051. https://doi.org/10.1016/j.ibiod.2020. 105051

Luo J, Gao Y, Tan K, Wei W, Liu X (2016) Preparation of a magnetic molecularly imprinted graphene composite highly adsorbent for 4-nitrophenol in aqueous medium. ACS Sustain Chem Eng 4:3316-3326. https://doi.org/10. 1021/acssuschemeng.6b00367

Ma H, Xu Z, Wang W, Gao X, Ma H (2019) Adsorption and regeneration of leaf-based biochar for p-nitrophenol adsorption from aqueous solution. RSC Adv 9:39282-39293. https://doi.org/10.1039/c9ra07943b

Man X, Wu R, Jiang X, Xu S, Wang W (2015) Organo-montmorillonite supported titania nanocomposite synthesized by using poly(methyl methacrylate) grafted cellulose as template and its application in photodegradation. Cellulose 22:3189-3198. https://doi.org/10.1007/s10570-015-07041

Mehdinia A, Dadkhah S, Kayyal TB, Jabbari A (2014) Design of a surface-immobilized 4-nitrophenol molecularly 
imprinted polymer via pre-grafting amino functional materials on magnetic nanoparticles. J Chromatogr A 1364:12-19. https://doi.org/10.1016/j.chroma.2014.08. 058

Mei Q, Zhang F, Wang N, Yang Y, Wu R, Wang W (2019) $\mathrm{TiO} 2 / \mathrm{Fe} 2 \mathrm{O} 3$ heterostructures with enhanced photocatalytic reduction of $\mathrm{Cr}(\mathrm{vi})$ under visible light irradiation. RSC Adv 9:22764-22771. https://doi.org/10.1039/ C9RA03531A

Meng J, Ren M, Wang S, Gao J, Shan X, Hu J (2020) A smart adsorbent with ability of environmentally friendly regeneration for p-nitrophenol removal in aqueous solution. J Inorg Organomet Polym. https://doi.org/10.1007/s10904020-01792-3

Miao H, Song S, Chen H, Zhang W, Han R, Yang G (2020) Adsorption study of p-nitrophenol on a silver (I) triazolate MOF. J Porous Mat 27:1409-1417. https://doi.org/10. 1007/s10934-020-00917-w

Narkkun T, Boonying P, Yuenyao C, Amnuaypanich S (2019) Green synthesis of porous polyvinyl alcohol membranes functionalized with 1-arginine and their application in the removal of 4-nitrophenol from aqueous solution. J Appl Polym Sci 136:47835. https://doi.org/10.1002/app.47835

Pan B et al (2007) Improved adsorption of 4-nitrophenol onto a novel hyper-cross-linked polymer. Environ Sci Technol 41:5057-5062. https://doi.org/10.1021/es070134d

Pang X, He Y, Jung J, Lin Z (2016) 1D nanocrystals with precisely controlled dimensions, compositions, and architectures. Science 353:1268-1272

Peydayesh M, Suter MK, Bolisetty S, Boulos S, Handschin S, Nystrom L, Mezzenga R (2020) Amyloid fibrils aerogel for sustainable removal of organic contaminants from water. Adv Mater 32:e1907932. https://doi.org/10.1002/adma. 201907932

Pichon V, Delaunay N, Combès A (2019) Sample preparation using molecularly imprinted polymers. Anal Chem 92:16-33. https://doi.org/10.1021/acs.analchem.9b04816

Rong Y, Han R (2019) Adsorption of p-chlorophenol and p-nitrophenol in single and binary systems from solution using magnetic activated carbon Korean. J Chem Eng 36:942-953. https://doi.org/10.1007/s11814-019-0267-1

Ronglan Wu LT, Wang W, Man X (2015) Bifunctional cellulose derivatives for the removal of heavy-metal ions and phenols: synthesis and adsorption studies. J Appl Polym Sci 132:41830. https://doi.org/10.1002/app.41830

Say R, Ersöz A, Şener İ, Atılır A, Diltemiz S, Denizli A (2005) Comparison of adsorption and selectivity characteristics for 4-nitrophenol imprinted polymers prepared via bulk and suspension polymerization. Sep Sci Technol 39:3471-3484. https://doi.org/10.1081/SS-200028939

Schirhagl R (2014) Bioapplications for molecularly imprinted polymers. Anal Chem 86:250-261. https://doi.org/10. $1021 / \mathrm{ac} 401251 \mathrm{j}$

Shen H-M et al (2015) Fast adsorption of p-nitrophenol from aqueous solution using $\beta$-cyclodextrin grafted silica gel. Appl Surf Sci 356:1155-1167. https://doi.org/10.1016/j. apsusc.2015.08.203

Sun Y, Wang B (2011) Preparation of a novel phthalimidomethylated polysulfone/unmodified polysulfone blend affinity membrane and applications for removal of p-nitrophenol. J Appl Polym Sci 122:1472-1479. https:// doi.org/10.1002/app.34124

Tan Z, Gao M, Dai J, Ge W, Zhu W, Yan Y (2020) Magnetic interconnected macroporous imprinted foams for selective recognition and adsorptive removal of phenolic pollution from water. Fibers Polym 21:762-774. https://doi.org/10. 1007/s12221-020-8695-4

Tian L et al (2018) A novel GO/PNIPAm hybrid with two functional domains can simultaneously effectively adsorb and recover valuable organic and inorganic resources. Chem Eng J343:607-618. https://doi.org/10.1016/j.cej. 2018.03.015

Velempini T, Pillay K, Mbianda XY, Arotiba OA (2019) Carboxymethyl cellulose thiol-imprinted polymers: synthesis, characterization and selective $\mathrm{Hg}(\mathrm{II})$ adsorption. J Environ Sci 79:280-296. https://doi.org/10.1016/j.jes.2018.11.022

Wu R, Tian L, Wang W (2015) Synthesis of a nanocomposite of organo-montmorillonite /cellulose-g-poly(methyl methacrylate) by atom-transfer radical polymerization and its application in removal of 2,4-dichlorophenol. Cellulose 22:3633-3643. https://doi.org/10.1007/s10570-015-07517

Wu Y et al (2020) Double-layer-based molecularly imprinted membranes for template-dependent recognition and separation: an imitated core-shell-based synergistic integration design. Che Eng J 398:125371. https://doi.org/10.1016/j. cej.2020.125371

Xiaolin Man RW, Lv H, Wang W (2015) Synthesis of a montmorillonite-supported titania nanocomposite withgrafted cellulose as a template and its application in photocatalyticdegradation. J Appl Polym Sci 132:42627. https:// doi.org/10.1002/app.42627

Yoshikawa M, Tharpa K, St-O D (2016) Molecularly imprinted membranes: past, present, and future. Chem Rev 116:11500-11528. https://doi.org/10.1021/acs.chemrev. $6 \mathrm{~b} 00098$

Yu M, Gao M, Shen T, Wang J (2018) Organo-vermiculites modified by low-dosage Gemini surfactants with different spacers for adsorption toward p-nitrophenol. Colloids Surf A Physicochem Eng 553:601-611. https://doi.org/10.1016/ j.colsurfa.2018.05.095

Zhang H, Li Y, Zheng D, Cao S, Chen L, Huang L, Xiao H (2019) Bio-inspired construction of cellulose-based molecular imprinting membrane with selective recognition surface for paclitaxel separation. Appl Surf Sci 466:244-253. https://doi.org/10.1016/j.apsusc.2018.10. 038

Zhang C, Shi X, Yu F, Quan Y (2020) Preparation of dummy molecularly imprinted polymers based on dextran-modified magnetic nanoparticles $\mathrm{Fe} 3 \mathrm{O} 4$ for the selective detection of acrylamide in potato chips. Food Chem 317:126431. https://doi.org/10.1016/j.foodchem.2020. 126431

Zheng $\mathrm{H}$ et al (2017) Adsorption of p-nitrophenols (PNP) on microalgal biochar: analysis of high adsorption capacity and mechanism. Bioresour Technol 244:1456-1464. https://doi.org/10.1016/j.biortech.2017.05.025

Zheng X, Zhang Y, Bian T, Wang D, Li Z (2019) One-step fabrication of imprinted mesoporous cellulose nanocrystals films for selective separation and recovery of $\mathrm{Nd}(\mathrm{III})$. 
Cellulose 26:5571-5582. https://doi.org/10.1007/s10570019-02482-1

Zhou J, Wang Y, Ma Y, Zhang B, Zhang Q (2019) Surface molecularly imprinted thermo-sensitive polymers based on light-weight hollow magnetic microspheres for specific recognition of BSA. Appl Surf Sci 486:265-273. https:// doi.org/10.1016/j.apsusc.2019.04.159

Zhou Z et al (2020) Preparation of highly efficient ion-imprinted polymers with $\mathrm{Fe}_{3} \mathrm{O}_{4}$ nanoparticles as carrier for removal of $\mathrm{Cr}(\mathrm{VI})$ from aqueous solution. Sci Total Environ 699:134334. https://doi.org/10.1016/j.scitotenv.2019. 134334

Publisher's Note Springer Nature remains neutral with regard to jurisdictional claims in published maps and institutional affiliations. 